\section{GW23-e2690 ARE THE LEFT VENTRICULAR FALSE TENDONS CONNECTING THE BASAL IVS TO THE APICAL OF LEFT VENTRICULAR FREE WALL NORMAL ANATOMIC STRUCTURES?}

doi:10.1136/heartjnl-2012-302920d.22

Caie Wu, Yifang Guo, Yongyi Bai, Peng Gao, Zhixin Ji, Caie Wu. Chinese PLA General Hospital

Objectives The echo cardiographic detection rates of the left ventricular false tendons (LVFTs) vary widely. Several diseases such as repolarisation abnormal, were relevant to LVFTs. The development of new technology has improved the detection of LVFTs and resolution, It showed that LVFTs may be more popular than ever reported. Nevertheless, the reports of epidemiology of location of LVFTs was few. In this study, we try to investigate the distribution of LVFTs locations in healthy population and hypertensive patients so that make clear which location be the most popular.

Methods From 1999 to 2005, we prospectively studied 1475 participants total including 1038 healthy subjects and 437 hypertensive patients. Every subject was examined using echocardiography with second harmonic imaging. The attachments of the LVFTs on ventricular wall were recorded. We visually measured the included angle formed by the interventricular septum and the LVFTs and classified the LVFTs accordingly as longitudinal $\left(\leq 45^{\circ}\right)$ and transverse $\left(>45^{\circ}\right)$ types. The $\chi^{2}$ test was used to compare the rates of LVFTs between groups.

Results Total of 1264 LVFTs were present in 1177 (79.8\%) of 1475 participants. Of 1264 LVFTS identified in 1177 participants, There were no significant differences between males and females in the detection rates of LVFTs between males and females (597/749 vs $580 / 746$, respectively; $p>0.05$ ), also between healthy subjects and hypertensive patients. The most common connection of the LVFTs was connecting the basal intraventricular septum to the apical left ventricular free wall (1094/1264, 86.6\%).

Conclusions LVFTs are commonly visualised using echocardiography. More than four locations of LVFTs were observed. The most common connection of the LVFTs was connecting the basal intraventricular septum to the apical left ventricular free wall. It suggested that the LVFTs at such location may be normal anatomic structures in the left ventricular cavity. The result will be helpful for us to investigate the distribution of LVFTs attachments in other repolarisation diseases in further studies 